\title{
Toxicidad de extracto acuoso de Stevia rebaudiana en Rattus norvegicus variedad Sprague Dawley
}

Toxicity of aqueous extract of Stevia rebaudiana in Rattus norvegicus variety Sprague Dawley

\author{
Julio Alberto Todco Palza y Henry Diaz-Murillo
}

Universidad Nacional de San Agustín. Arequipa Perú.

\section{INFORMACIÓN}

\section{Historia del Artículo \\ Recepción: 12/02/2019 \\ Revisión: 03/04/2019}

Aceptación: 15/04/2019

\section{Palabras Clave}

Stevia rebaudiana, Toxicidad, Steviosidos.

\section{Key Words}

Stevia rebaudiana, Toxicity, Steviosides.

\section{DOI}

https://doi.org/10.35286/veritas. v20i1.235

\begin{abstract}
RESUMEN
Se evaluó la toxicidad del extracto acuoso de Stevia rebaudiana en Rattus norvegicus variedad Sprague Dawley durante 60 días. Se llevó a cabo en el Bioterio de Biología UNSA, Arequipa, durante los meses Agosto a Diciembre del 2012. Se utilizaron 16 Rattus norvegicus variedad Sprague Dawley distribuidas en cuatro grupos de 4 ratas cada uno: Al grupo control se le administró agua destilada (Grupo Control), al grupo experimental 2, 3, 4 se le administró vía orogástrica extracto acuoso de Stevia rebaudiana a dosis de $0.3,0.6$ y $0.9 \mathrm{~g} / \mathrm{Kg}$./día respectivamente. Se utilizó los kit de Wierner para la determinación de TGO, TGP, glucosa y creatinina. El peso corporal se midio en una balanza digital Omni 5300. Los tejidos evaluados (hígado, riñón y estomago) por el método de Harris. No se encontró diferencia significativa en la actividad de TGO y TGP en plasma de Rattus norvegicus variedad Sprague Dawley con tratamiento de extracto acuoso de Stevia rebaudiana en dosis de $0.3,0.6$ y $0.9 \mathrm{~g} / \mathrm{kg} /$ día durante 60 dias $(\mathrm{p}>0.05)$. Se encontró disminución significativa en los niveles de glucosa en plasma de Rattus norvegicus variedad Sprague Dawley con tratamiento de extracto acuoso de Stevia rebaudiana en dosis de $0.3,0.6$ y $0.9 \mathrm{~g} / \mathrm{kg} /$ día durante 60 dias ( $>00.05$ ). No se observó diferencia significativa en los niveles de creatinina en los tratamientos con extracto acuoso de Stevia rebaudiana 0.3, 0.6 y $0.9 \mathrm{~g} / \mathrm{kg} /$ día respecto al grupo control ( $>0.05$ ). Se encontró una disminución significativa en el peso corporal de las ratas con tratamiento con extracto acuoso de Stevia rebaudiana 0.3, 0.6 y 0.9 $\mathrm{g} / \mathrm{kg} /$ día respecto al grupo control $(\mathrm{p}<0.05)$. No se encontró alteración microscópica en Higado, riñón y estomago de Rattus norvegicus variedad Sprague Dawley con tratamiento de extracto acuoso de Stevia rebaudiana en dosis de 0.3, 0.6 y $0.9 \mathrm{~g} / \mathrm{kg} /$ día durante 60 días.
\end{abstract}

\begin{abstract}
The toxicity of the aqueous extract of Stevia rebaudiana in Rattus norvegicus variety Sprague Dawley was evaluated for 60 days. It was held at the Biology Biology UNSA, Arequipa, during the months August to December 201216 Rattus norvegicus Sprague Dawley variety distributed in four groups of 4 rats each were used: The distilled water (Control Group) was administered to the control group, the Stevia rebaudiana aqueous extract was administered to the orogastric group experimentally $2,3,4$ doses of $0.3,0.6$ and $0.9 \mathrm{~g} / \mathrm{kg} /$ day respectively. Wierner's kits were used for the determination of TGO, TGP, glucose and creatinine. Body weight was measured on an Omni 5300 digital scale. The tissues evaluated (liver, kidney and stomach) by the Harris method. There was no significant difference in the activity of TGO and TGP in plasma of Rattus norvegicus variety Sprague Dawley with treatment of aqueous extract of Stevia rebaudiana in doses of 0.3, 0.6 and $0.9 \mathrm{~g} / \mathrm{kg} /$ day for 60 days ( $\mathrm{p}>0.05$ ). There was a significant decrease in plasma glucose levels of Rattus norvegicus Sprague Dawley variety with treatment of aqueous extract of Stevia rebaudiana in doses of $0.3,0.6$ and $0.9 \mathrm{~g} / \mathrm{kg} /$ day for 60 days $(\mathrm{p}>0.05)$. There was no significant difference in creatinine levels in treatments with aqueous extract of Stevia rebaudiana 0.3, 0.6 and $0.9 \mathrm{~g} / \mathrm{kg} /$ day with respect to the control group $(\mathrm{p}>0.05)$. A significant decrease was found in the body weight of the rats treated with aqueous extract of Stevia rebaudiana $0.3,0.6$ and 0.9 $\mathrm{g} / \mathrm{kg} /$ day with respect to the control group $(\mathrm{p}<0.05)$. No microscopic alteration was found in Liver, kidney and stomach of Rattus norvegicus Sprague Dawley variety with treatment of aqueous extract of Stevia rebaudiana in doses of $0.3,0.6$ and $0.9 \mathrm{~g} / \mathrm{kg} /$ day for 60 days.
\end{abstract}

\section{INTRODUCCIÓN}

El Steviosido, un componente abundante de la hoja de Stevia rebaudiana, se ha hecho famoso por su intenso dulzor (250-300 veces más dulce que la sacarosa) y está siendo usado como un dulcificante no calórico en diferentes países. Varios estudios han sugerido que, además del dulzor, el

Correspondencia:

Henry Díaz-Murillo

hdiazmur@gmail.com steviosido junto con sus compuestos relacionados, entre los que se incluyen rebaudiosido (el segundo componente más abundante de la hoja de S. rebaudiana), steviol e isosteviol (componentes metabólicos del steviosido) también ofrecen beneficios terapéuticos, debido a que contienen antihiperglicemicos, anti-hipertensivos, anti-inflammatorios, antitumorales, anti-diarreicos, diuréticos, y acciones inmunomoduladoras. Cabe resaltar que sus efectos a nivel de la glucosa en el plasma y tensión arterial sólo son observados cuando estos parámetros son más altos de lo normal. Puesto que el steviol puede actuar como transportadores de 
moléculas, se propone como un modulador de fármacos.

Por lo anteriormente mencionado es que nos proponemos evaluar la toxicidad de Stevia rebauidina en forma crónica via oral en Rattus norvegicus Variedad Sprague Dawley.

Debido a su uso popular como sustituto del azúcar no calórico, han sido estudiadas de manera intensiva las propiedades toxicológicas del steviosido y el steviol tanto en condiciones in vitro como en animales experimentales. Además, se han evaluado los potenciales carcinogénicos y teratogénicos así como los efectos en la reproducción.

\section{MATERIAL Y METODOS}

\section{MATERIALES}

\section{a) Material Biológico}

- Stevia rebaudiana.

- Rattus norvegicus variedad Sprague Dawley.

- Mus musculus

\section{b) Equipos}

- Balanza analítica.

- Centrífuga.

- Cocina eléctrica.

- Espectrofotómetro.

- Estufa.

- Equipo de disección.

- Microcentrífuga.

- Microscopio.

- Licuadora.

\section{MÉTODOLOGIA}

\section{MÉTODO PARA TRANSAMINASAS TGO TGP}

La muestra de sangre se obtendrá por el método de Dorman extraído de la punta de la cola del animal de experimentación con la ayuda de un tubo capilar.

\section{Fundamento}

- La TGP cataliza la siguiente reacción: L- alanina + a-cetoglutarato TGP Glutamato + Piruvato.

- La TGO cataliza la siguiente reacción: L-aspartato + a-cetoglutarato TGO Glutamato + Oxalacetato

El piruvato formado (el oxalacetato es inestable $\mathrm{y}$ se transforma en piruvato) reacciona con la 2,4-dinitrofenilhidrazina produciendo en medio alcalino un compuesto coloreado que se mide a $505 \mathrm{~nm}$.

Se utilizará el método colorimétrico. (Reitman y Frankel) para la determinación de la actividad transaminasa glutámico pirúvica (TGP) y transaminasa glutámico oxalacética (TGO) en suero.

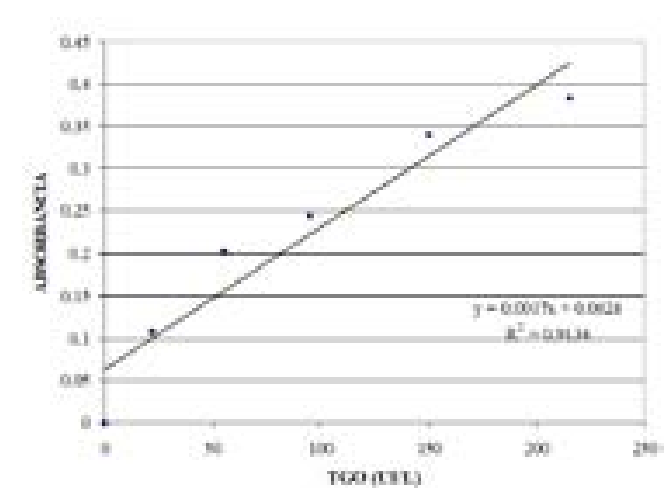

CURVA DE CALIBRACIÓN DE TGO (UI/L)

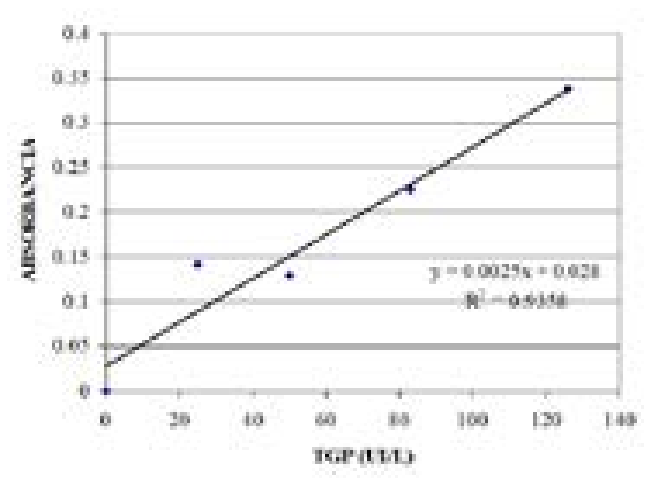

CURVA DE CALIBRACIÓN DE TGP(UI/L)

\section{MÉTODO DETERMINACIÓN GLUCOSA: Método Wiener}

El esquema de reacción es el siguiente:

GOD

Glucosa + $02+$ H20 > ácido glucónico + H202

POD

$\mathrm{H} 202+4-\mathrm{AF}+4$-hidroxibenzoato > quinonimina roja PROCEDIMIENTO

En tres tubos de fotocolorímetro marcados con B (Blanco) S (Standard) y D (Desconocido) colocar:

\begin{tabular}{lcccc}
\hline & & B & S & D \\
\hline Standard & - & $20 \mathrm{ul}$ & - & \\
Muestra & - & - & $20 \mathrm{ul}$ & \\
\multicolumn{2}{l}{ Reactivo de Trabajo } & $2 \mathrm{ml}$ & $2 \mathrm{ml}$ & $2 \mathrm{ml}$ \\
\hline
\end{tabular}

Incubar 5 minutos en baño de agua a $37^{\circ} \mathrm{C}$. Luego leer en espectrofotómetro a $505 \mathrm{~nm}$, llevando el aparato a cero con el blanco.

\section{MÉTODO DETERMINACIÓN DE CREATININA: Método de Wiener}

- Longitud de onda: $510 \mathrm{~nm}$ en espectrofotómetro

- Temperatura de reacción: $37^{\mathrm{a}} \mathrm{C}$.

- Tiempo de reacción: 15 minutos.

- Volumen de muestra: $0.1 \mathrm{ml}$

- Volumen de Reactivo: $1 \mathrm{ml}$

- Volumen final de reacción: $1.15 \mathrm{ml}$. 


\section{PROCEDIMIENTO}

En tres tubos marcados B (blanco), S (Standard) y D (desconocido) colocar:

\begin{tabular}{cccc}
\hline & B & S & D \\
\hline Agua destilada & $100 \mathrm{ul}$ & $-\cdots--$ & --- \\
Standard & ---- & $100 \mathrm{ul}$ & ---- \\
Muestra & ---- & $-\cdots--$ & $100 \mathrm{ul}$ \\
Reactivo de trabajo & $1 \mathrm{ml}$ & $1 \mathrm{ml}$ & $1 \mathrm{ml}$ \\
\hline
\end{tabular}

Mezclar. Incubar 10 minutos en baño Maria a 37o C. Dentro de los 15 minutos de retirado del baño, leer el Standard y el desconocido (D1) en espectrofotómetro a $510 \mathrm{~nm}$ llevando a 0.0 D.O. con el blanco. Luego agregar:

$$
\begin{array}{llll}
\text { Stopper } & 50 \mathrm{ul} & --- & 50 \mathrm{ul}
\end{array}
$$

Mezclar. Dejar los tubos 5 minutos a temperatura ambiente y volver a leer el Desconocido (D2), llevando a 0.0 D.O. con el blanco.

El color de la reacción es estable durante 6 horas por lo que la absorbancia debe ser leída dentro de ese lapso.

\section{CALCULO DE LOS RESULTADOS}

$$
\begin{gathered}
\text { Creatinina en suero }(\mathbf{m g} / \mathbf{l})=(\mathbf{D} 1-\mathbf{D} 2) \times \mathbf{~ f} \\
\mathrm{f}=20 \mathrm{mg} / \mathrm{l}
\end{gathered}
$$$$
\mathrm{S}
$$

\section{EVALUACIÓN HISTOLÓGICA MÉTODO DE HARRIS}

Fue realizado de acuerdo al método de Harris

\section{Diagnóstico Histopatológico}

El Diagnóstico Histopatológico de las muestras obtenidas se realizó en el laboratorio de Anatomía Patológia del Hospital CASE ESSALUD por el Dr. Henry Mercado.

\section{MÉTODO ESTADÍSTICO}

Los datos se expresaron como promedios. Se aplicó Análisis de Varianza (ANOVA) para establecer las diferencias significativas $(\mathrm{p}<0.01)$ en los promedios de TGO, TGP, Glucosa y creatinina y peso de las ratas en los diferentes tratamientos evaluados y se aplicó la prueba de especificidad de Tukey (P:0.05) para establecer el mejor tratamiento para el efecto del extracto acuoso de Stevia rebaudiana en Rattus norvegicus variedad Sprague Dawley. Se utilizó el paquete estadístico computarizado SPSS versión 18 para Windows 7 .

\section{RESULTADOS}

Se muestran los resultados en Tablas, gráficos y Figuras del efecto del extracto acuoso de Stevia rebaudiana sobre rattus norvegicus variedad Sprague Dawley por 60 días
Tabla 1: VALORES PROMEDIO DE TGO (UI/L) EN RATTUS NORVEGICUS VARIEDAD SPRAGUE DAWLEY SEGÚN TRATAMIENTO CON EXTRACTO ACUOSO DE Stevia rebaudiana

\begin{tabular}{ccccc}
\hline \multirow{2}{*}{ TRATAMIENTOS } & \multicolumn{4}{c}{ TGO (UI/L) } \\
\cline { 2 - 5 } CONTROL & BASAL & 30 & 45 & 60 \\
$\begin{array}{c}\text { Stevia rebaudiana } \\
0.3 \mathrm{~g} / \mathrm{kg} / \text { día }\end{array}$ & 60.10 & 75.01 & 74.99 & 79.76 \\
$\begin{array}{c}\text { Stevia rebaudiana } \\
0.6 \mathrm{~g} / \mathrm{kg} / \text { día }\end{array}$ & 65.92 & 70.28 & 75.51 & 72.84 \\
$\begin{array}{c}\text { Stevia rebaudiana } \\
0.9 \mathrm{~g} / \mathrm{kg} / \text { día }\end{array}$ & 66.16 & 72.76 & 71.35 & 72.08 \\
\hline F (ANOVA) & 1.71 & 0.83 & 0.30 & 1.83 \\
P & $>0.05$ & $>0.05$ & $>0.05$ & $>0.05$ \\
SIGNIFICANCIA & NS & NS & NS & NS \\
\hline
\end{tabular}

\section{abcTUKEY $(\mathrm{p}<0.05)$}

En la Tabla y Gráfico $\mathrm{N}^{\circ} 1$ se observa que los valores basales no muestran diferencia significativa entre los tratamientos fluctuando los promedios de $60.10 \mathrm{UI} / \mathrm{L}$ a 67.51 UI/L. A los 60 dias no se evidencia diferencia significativa en los tratamientos con extracto acuoso de Stevia rebaudiana $0.3,0.6$ y $0.9 \mathrm{~g} / \mathrm{kg} / \mathrm{dá}$ respecto al grupo control $(\mathrm{p}<0.05)$. Por lo que podemos establecer que el extracto acuoso de Stevia rebaudina no induce insuficiencia cardiaca.

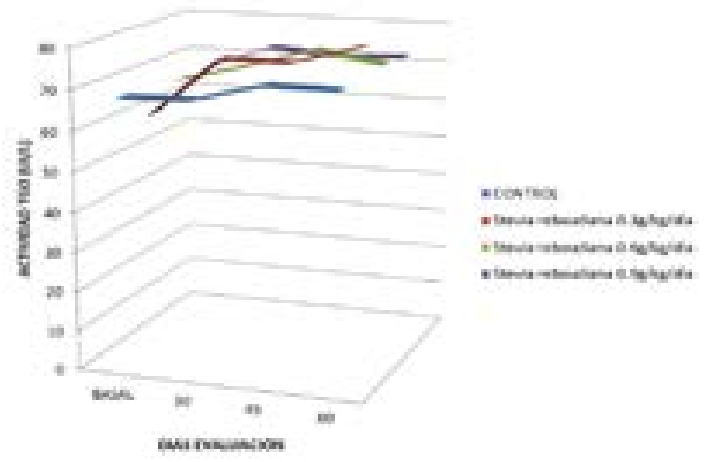

Graf 1: VALORES PROMEDIO DE TGO (UI/L) EN RATTUS NORVEGICUS VARIEDAD SPRAGUE DAWLEY SEGÚN TRATAMIENTO CON EXTRACTO ACUOSO DE Stevia rebaudiana

Tabla 2: VALORES PROMEDIO DE TGP (UI/L) EN RATTUS NORVEGICUS VARIEDAD SPRAGUE DAWLEY SEGÚN TRATAMIENTO CON EXTRACTO ACUOSO DE Stevia rebaudiana

\begin{tabular}{ccccc}
\hline \multirow{2}{*}{ TRATAMIENTOS } & \multicolumn{4}{c}{ TGP $(\mathrm{UI} / \mathrm{L})$} \\
\cline { 2 - 5 } CONTROL & BASAL & 30 & 45 & 60 \\
\hline Stevia rebaudiana $0.3 \mathrm{~g} / \mathrm{kg} / \mathrm{dí} a$ & 56.26 & 56.32 & 57.53 & 55.36 \\
Stevia rebaudiana $0.6 \mathrm{~g} / \mathrm{kg} /$ día & 57.61 & 57.38 & 52.35 & 56.30 \\
Stevia rebaudiana 0.9g/kg/día & 57.17 & 53.54 & 57.51 & 57.24 \\
\hline F (ANOVA) & 0.27 & 2.33 & 1.77 & 0.53 \\
P & $>0.05$ & $>0.05$ & $>0.05$ & $>0.05$ \\
SIGNIFICANCIA & NS & NS & NS & NS \\
\hline
\end{tabular}

${ }^{a b c}$ TUKEY $(p<0.05)$ 
En la Tabla y Gráfico $\mathrm{N}^{\circ} 2$ se observa que los valores basales no muestran diferencia significativa entre los tratamientos fluctuando los promedios de 55.26 UI/L a 57.61 UI/L. A los 60 dias no se evidencia diferencia significativa en los tratamientos con extracto acuoso de Stevia rebaudiana $0.3,0.6$ y $0.9 \mathrm{~g} / \mathrm{kg} /$ día respecto al grupo control $(\mathrm{p}<0.05)$. Por lo que podemos establecer que el extracto acuoso de Stevia rebaudina no induce insuficiencia hepática.

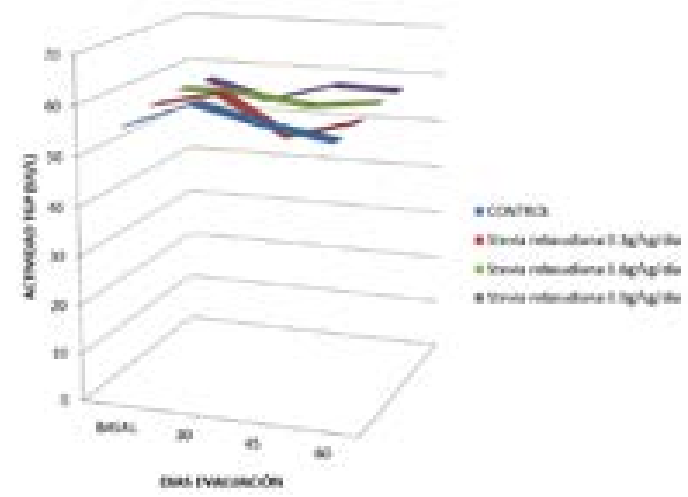

Graf 2: VALORES PROMEDIO DE TGP (UI/L) EN RATTUS NORVEGICUS VARIEDAD SPRAGUE DAWLEY SEGÚN TRATAMIENTO CON EXTRACTO ACUOSO DE Stevia rebaudiana

Tabla 3: VALORES PROMEDIO DE GLUCOSA (mg/ dI) EN RATTUS NORVEGICUS VARIEDAD SPRAGUE DAWLEY SEGÚN TRATAMIENTO CON EXTRACTO ACUOSO DE Stevia rebaudiana

\begin{tabular}{ccccc}
\hline \multirow{2}{*}{ TRATAMIENTOS } & \multicolumn{4}{c}{ GLUCOSA (mg/dl) } \\
\cline { 2 - 5 } & BASAL & 30 & 45 & 60 \\
\hline $\begin{array}{c}\text { CONTROL } \\
\text { Stevia rebaudiana 0.3g/ } \\
\text { kg/día }\end{array}$ & 117.00 & 106.75 & 100.25 & $99.69 \mathrm{~b}$ \\
$\begin{array}{c}\text { Stevia rebaudiana 0.6g/ } \\
\text { kg/día }\end{array}$ & 120.5 & 120.50 & 105.50 & $96.75 \mathrm{a}$ \\
$\begin{array}{c}\text { Stevia rebaudiana 0.9g/ } \\
\text { kg/día }\end{array}$ & 116.25 & 119.25 & 103.25 & $95.00 \mathrm{a}$ \\
\hline $\begin{array}{c}\text { F (ANOVA) } \\
\text { P }\end{array}$ & 1.85 & 3.44 & 2.31 & 4.57 \\
SIGNIFICANCIA & NS & NS & NS & S \\
\hline
\end{tabular}

${ }^{\mathrm{abc} T U K E Y}(\mathrm{p}<0.05)$

En la Tabla y Gráfico $N^{\circ} 3$ se observa que los valores basales no muestran diferencia significativa entre los tratamientos fluctuando los promedios de 116.25 a 122.25 $\mathrm{mg} / \mathrm{dl} \quad(\mathrm{p}>0.05)$. A los 60 dias se evidencia disminución significativa en los tratamientos con extracto acuoso de Stevia rebaudiana $0.3,0.6$ y $0.9 \mathrm{~g} / \mathrm{kg} /$ día respecto al grupo control $(\mathrm{p}<0.05)$. Por lo que podemos establecer que el extracto acuoso de Stevia rebaudina disminuye los niveles de glicemia en comparación al grupo control.

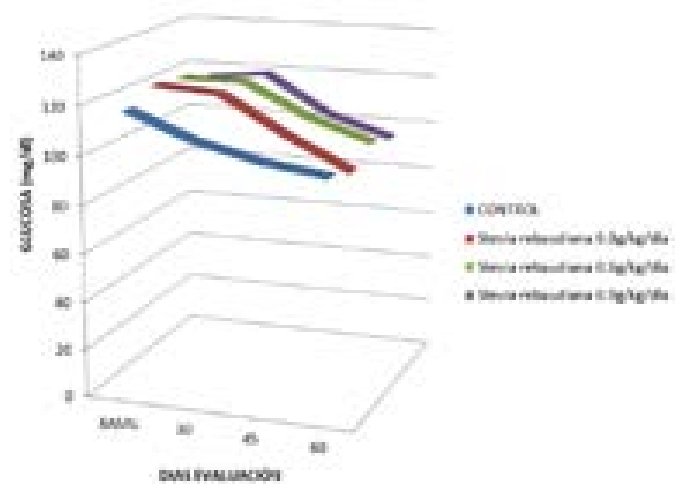

Graf 3: VALORES PROMEDIO DE GLUCOSA (mg/dl) EN RATTUS NORVEGICUS VARIEDAD SPRAGUE DAWLEY SEGÚN TRATAMIENTO CON EXTRACTO ACUOSO DE Stevia rebaudiana

Tabla 4: VALORES PROMEDIO DE CREATININA (mg/ dI) EN RATTUS NORVEGICUS VARIEDAD SPRAGUE DAWLEY SEGÚN TRATAMIENTO CON EXTRACTO ACUOSO DE Stevia rebaudiana.

\begin{tabular}{ccccc}
\hline \multirow{2}{*}{ TRATAMIENTOS } & \multicolumn{4}{c}{ CREATININA (mg/dl) } \\
\cline { 2 - 5 } & BASAL & 30 & 45 & 60 \\
\hline $\begin{array}{c}\text { CONTROL } \\
\text { Stevia rebaudiana 0.3g/ } \\
\text { kg/día }\end{array}$ & 0.60 & 0.59 & 0.60 & 0.63 \\
$\begin{array}{c}\text { Stevia rebaudiana 0.6g/ } \\
\text { kg/día }\end{array}$ & 0.64 & 0.63 & 0.62 & 0.62 \\
$\begin{array}{c}\text { Stevia rebaudiana 0.9g/ } \\
\text { kg/día }\end{array}$ & 0.65 & 0.64 & 0.65 & 0.61 \\
\hline $\begin{array}{c}\text { F (ANOVA) } \\
\text { P }\end{array}$ & 1.95 & 3.66 & 0.91 & 1.53 \\
SIGNIFICANCIA & NS & NS & NS & NS \\
\hline
\end{tabular}

${ }^{\text {abc TUKEY }(\mathrm{p}<0.05)}$

En la Tabla y Gráfico $N^{\circ} 4$ se observa que los valores basales no muestran diferencia significativa entre los tratamientos fluctuando los promedios de 0.6 a $0.65 \mathrm{mg} / \mathrm{dl}$ ( $p>0.05$ ). A los 60 dias no existe diferencia significativa en los tratamientos con extracto acuoso de Stevia rebaudiana $0.3,0.6$ y $0.9 \mathrm{~g} / \mathrm{kg} /$ día respecto al grupo control ( $\mathrm{p}>0.05)$. Por lo que podemos establecer que el extracto acuoso de Stevia rebaudina no provoca insuficiencia renal.

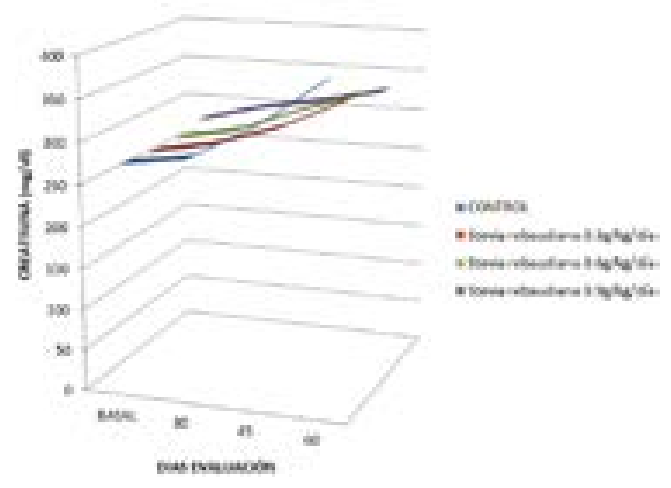

Graf 4: VALORES PROMEDIO DE CREATININA (mg/dl) EN RATTUS NORVEGICUS VARIEDAD SPRAGUE DAWLEY SEGÚN TRATAMIENTO CON EXTRACTO ACUOSO DE Stevia rebaudiana 
Tabla 5: VALORES PROMEDIO DE PESO CORPORAL (g) EN RATTUS NORVEGICUS VARIEDAD SPRAGUE DAWLEY SEGÚN TRATAMIENTO CON EXTRACTO ACUOSO DE Stevia rebaudiana

\begin{tabular}{ccccc}
\hline TRATAMIENTOS & \multicolumn{4}{c}{ PESO CORPORAL $(\mathrm{G})$} \\
\cline { 2 - 5 } & BASAL & 30 & 45 & 60 \\
\hline CONTROL & 272 & 284 & 326 & $383^{\mathrm{c}}$ \\
$\begin{array}{c}\text { Stevia rebaudiana } \\
0.3 \mathrm{~g} / \mathrm{kg} / \text { día }\end{array}$ & 269 & 284 & 309 & $346^{\mathrm{b}}$ \\
$\begin{array}{c}\text { Stevia rebaudiana } \\
0.6 \mathrm{~g} / \mathrm{kg} / \text { día }\end{array}$ & 269 & 285 & 315 & $340^{\mathrm{b}}$ \\
$\begin{array}{c}\text { Stevia rebaudiana } \\
0.9 \mathrm{~g} / \mathrm{kg} / \text { día }\end{array}$ & 278 & 298 & 312 & $329^{\mathrm{a}}$ \\
\hline F (ANOVA) & 0.64 & 4.18 & 5.31 & 74.32 \\
P & $>0.05$ & $>0.05$ & $<0.05$ & $<0.01$ \\
SIGNIFICANCIA & $\mathrm{NS}$ & $\mathrm{NS}$ & $\mathrm{S}$ & AS \\
\hline
\end{tabular}

${ }^{a b c}$ TUKEY $(p<0.05)$

En la Tabla y Gráfico $N^{\circ} 5$ se observa que los valores basales no muestran diferencia significativa entre los tratamientos fluctuando los promedios de 269 a 278 gramos ( $\mathrm{p}>0.05$ ). A los 60 dias se evidencia disminución significativa en los tratamientos con extracto acuoso de Stevia rebaudiana $0.3,0.6$ y $0.9 \mathrm{~g} / \mathrm{kg} /$ día respecto al grupo control $(\mathrm{p}<0.05)$.

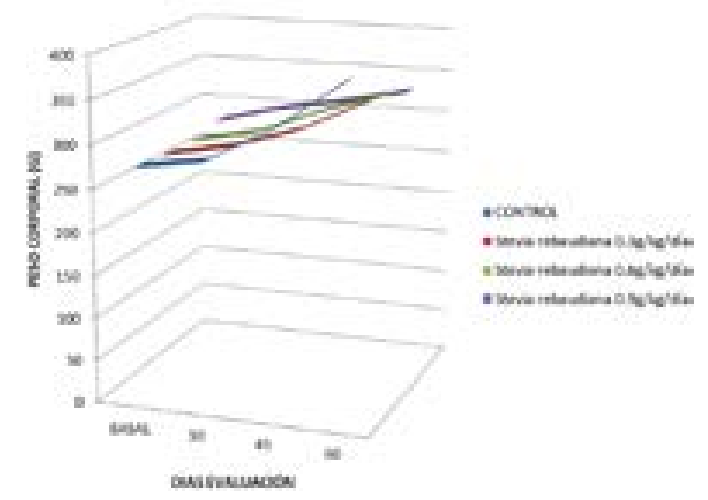

Graf 5: VALORES PROMEDIO DE PESO CORPORAL (g) EN RATTUS NORVEGICUS VARIEDAD SPRAGUE DAWLEY SEGÚN TRATAMIENTO CON EXTRACTO ACUOSO DE Stevia rebaudiana

\section{EVALUACIÓN HISTOLÓGICA}

A continuación mostramos fotografías de corte histológico de hígado de Rattus norvegicus variedad Sprague Dawley, con aumentos de $400 \mathrm{X}$ respectivamente.

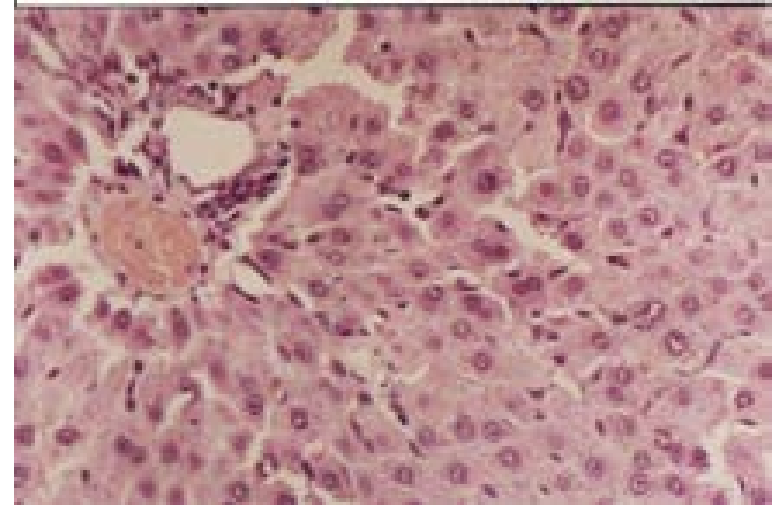

Fig 1: ESTRUCTURA DE HÍGADO DEL GRUPO CONTROL EN Rattus norvegicus variedad Sprague Dawley, (HEMATOXILINA EOSINA $400 \mathrm{X}$ ).

En esta microfotografía observamos la citoarquitectura normal de hígado de ratas del grupo control. Se puede observar:

1: Hepatocitos

2: Vasos sanguíneos

3: Porta hepatica

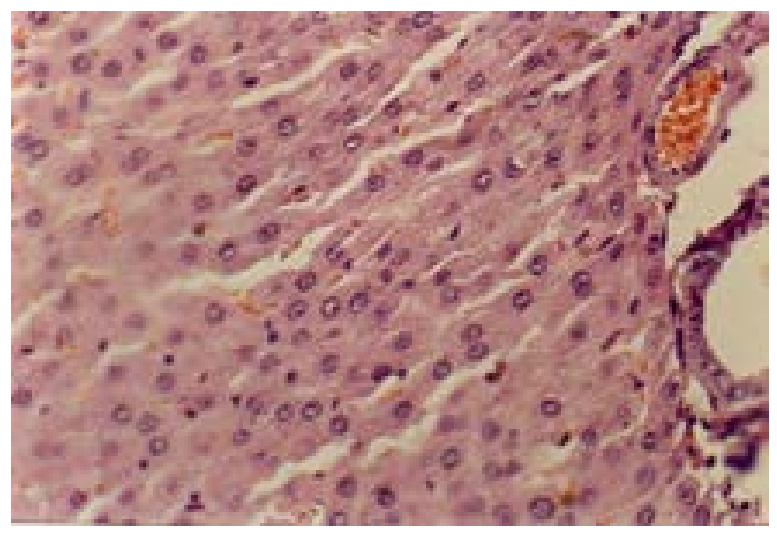

Fig 2: ESTRUCTURA DE HIGADO DE TRATAMIENTO EXTRACTO ACUOSO DE STEVIA REBAUDINA $0.9 \mathrm{~g} / \mathrm{kg} /$ día DE Rattus norvegicus VARIEDAD SPRAGUE DAWLEY POR 60 DIAS HEMATOXILINA EOSINA $400 \mathrm{X}$

En esta microfotografía observamos hígado de ratas del grupo con dosis de extracto acuoso de Stevia rebaudiana 0.9 $\mathrm{g} / \mathrm{kg} /$ día durante 60 días de tratamiento. Se puede observar estructura normal:

1: Hepatocitos

2: Vasos sanguíneos

3: Porta hepatica 


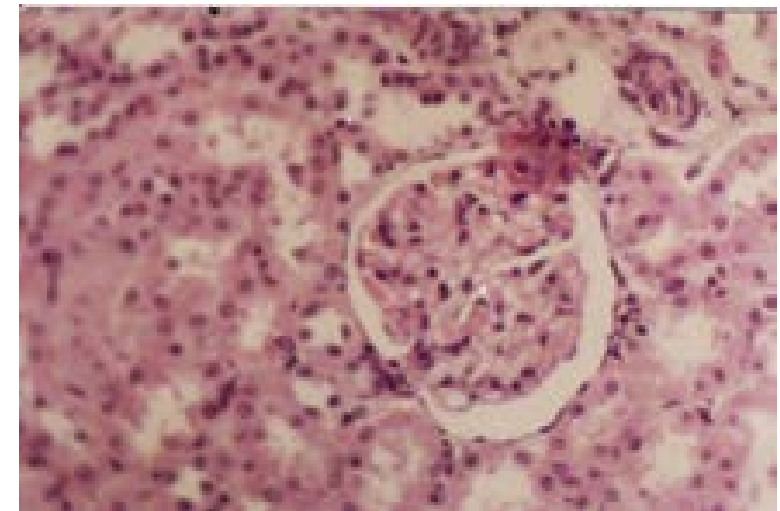

Fig 3: ESTUdio HISTOLOGICO DE RIÑON DE TRATAMIENTO DE GRUPO CONTROL DE Rattus norvegicus VARIEDAD SPRAGUE DAWLEY HEMATOXILINA EOSINA $400 \mathrm{X}$

En esta microfotografía observamos la citoarquitectura normal de riñón de ratas del grupo control. Se puede observar:

1: Capilares tubulares

2: Glomérulo de Malpighi

3: Capsula de Bowman

4: Túbulos colectores

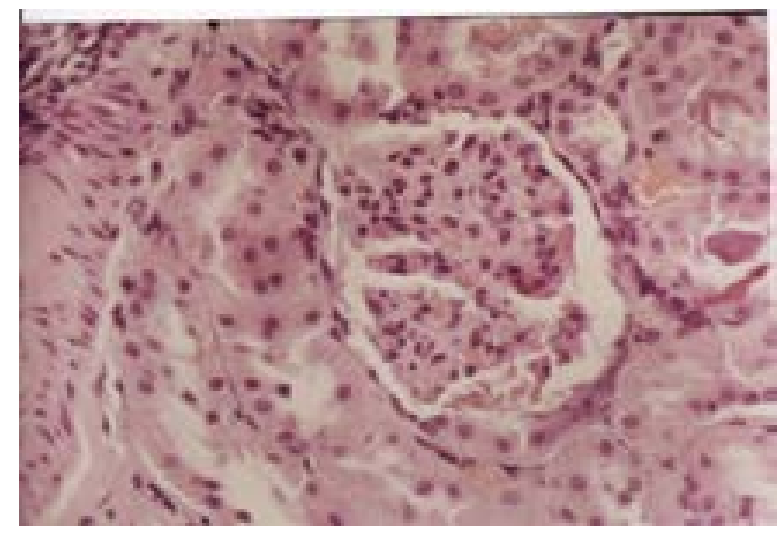

Fig 4: ESTRUCTURA DE RIÑON DE TRATAMIENTO EXTRACTO ACUOSO DE STEVIA REBAUDINA $0.9 \mathrm{~g} / \mathrm{kg} /$ día DE Rattus norvegicus VARIEDAD SPRAGUE DAWLEY POR 60 DIAS HEMATOXILINA EOSINA $400 \mathrm{X}$

En esta microfotografía observamos riñón de ratas del grupo con dosis de extracto acuoso de Stevia rebaudiana0.9 $\mathrm{g} / \mathrm{kg} /$ día durante 60 días de tratamiento. Se puede observar estructura normal:

1: Capilares tubulares

2: Glomérulo de Malpighi

3: Capsula de Bowman

4: Túbulos colectores

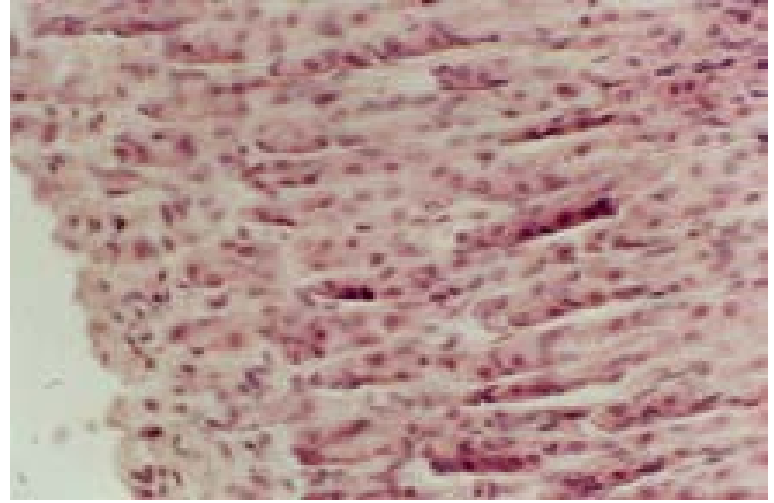

Fig 5: ESTRUCTURA DE ESTÓMAGO DE TRATAMIENTO CONTROL DE Rattus norvegicus VARIEDAD SPRAGUE DAWLEY HEMATOXILINA EOSINA $400 \mathrm{X}$

En esta microfotografía observamos la citoarquitectura normal de estómago de ratas del grupo control.

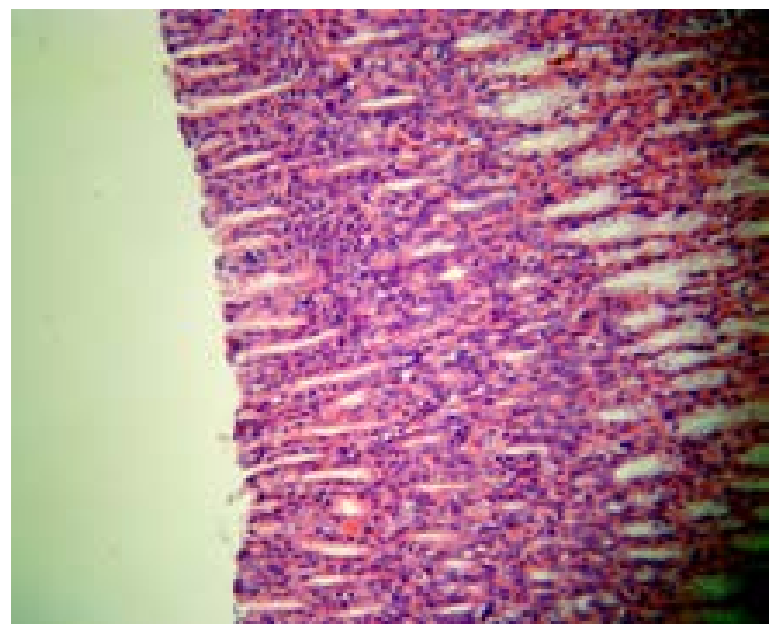

Fig 6: ESTRUCTURA DE ESTOMAgo DE TRATAMIENTO EXTRACTO ACUOSO DE STEVIA REBAUDINA $0.9 \mathrm{~g} / \mathrm{kg} / \mathrm{día}$ DE Rattus norvegicus VARIEDAD SPRAGUE DAWLEY POR 60 DIAS HEMATOXILINA EOSINA $400 \mathrm{X}$

En esta microfotografía de estómago observamos estructura dentro de los limites normales en ratas tratadas con extracto acuoso de Stevia rebaudiana en dosis de $0.9 \mathrm{~g} /$ $\mathrm{kg} /$ día.

\section{DISCUSION}

No se encontró diferencia significativa en la actividad de TGO y TGP en plasma de Rattus norvegicus variedad Sprague Dawley con tratamiento de extracto acuoso de Stevia rebaudiana en dosis de $0.3,0.6$ y $0.9 \mathrm{~g} / \mathrm{kg} /$ día durante 60 dias $(\mathrm{p}>0.05)$. Por lo que podemos establecer que el extracto acuoso de Stevia rebaudina no induce insuficiencia cardiaca y hepática. Estos resultados coinciden con lo reportado por (Xili et al., 1992; Toskulkao et al.,1997)

Se encontró disminución significativa en los niveles de glucosa en plasma de Rattus norvegicus variedad Sprague Dawley con tratamiento de extracto acuoso de Stevia rebaudiana en dosis de $0.3,0.6$ y $0.9 \mathrm{~g} / \mathrm{kg} /$ día durante 60 dias ( $>0.05$ ). Por lo que podemos establecer que el extracto acuoso de Stevia rebaudina disminuye los niveles de glicemia en comparación al grupo control. Estos resultados coinciden con lo reportado por (Jeppesen et al., 2000; Lailerd et al., 2004; JECFA,2006). 
Esta disminución de los niveles de glucosa se deberian probablemente a que la inhibición de la absorción de la glucosa por el steviol se debe a la reducción del contenido de ATP en la mucosa intestinal, lo que es una consecuencia de la disminución de las actividades enzimáticas mitocondriales en el intestino al nivel de fosforilación y alteraciones morfológicas de las células de absorción intestinales. De hecho, la inhibición por steviol de la absorción de glucosa en células intestinales podría resultar en disminución del nivel de glucosa plasmática, lo que sería indeseable en individuos sanos. De todas formas, la ingesta diaria aceptable (IDA) de steviosido $(5 \mathrm{mg} / \mathrm{Kg}$ de peso corporal/día) tendría un rendimiento máximo de concentración de steviol de aproximadamente $20 \mu \mathrm{M}$ si el steviosido se convierte por completo en steviol (JECFA,2006). Esta concentración de steviol esta muy por debajo de la cantidad estudiada para inhibir la absorción de glucosa intestinal.

Asimismo la reducción de la glicemia se deberia probablemente a que steviosido estimula la secreción de insulina (Jeppesen et al., 2000) y así como el aumento de la sensibilidad a la insulina (Lailerd et al., 2004) subrayan sus efectos beneficiosos sobre el metabolismo de la glucosa. En realidad, se observó una reducción en la glucosa plasmática junto con un incremento de la secreción de insulina, en las ratas diabéticas de tipo 2 de Goto-Kakizaki después de una ingesta oral de steviosido de $25 \mathrm{mg} / \mathrm{kg}$ de peso corporal $/$ día durante 6 semanas (Jeppesen et al., 2003).

Sin embargo, estos resultados aumenta la preocupación sobre si la ingesta de steviosido en ayunas puede conducir a la hipoglucemia, como en el la situación de sulfonilurea, que estimula la liberación de insulina, constituyendo así, una amenaza potencial para sujetos diabéticos Para contestar esta cuestión, se ha administrado steviosido a ratas GK y Wistar en ayunas, con una concentración normal y baja de glucosa plasmática (3,3 $\mathrm{mM}$ o menos), y no se encontró acción insulinotrópica (Jeppesen et al., 2002). De hecho, el steviosido estimula la liberación de insulina sólo en algunos casos aislados de ratones con altas concentraciones de glucosa (> 8,3 mM) (Jeppesen et al., 2000). De ahí, es probable que el steviosido muestre su efecto beneficioso estimulando la liberación de insulina sólo en estado diabético.

Se han realizado un número limitado de estudios experimentales para evaluar el efecto del extracto de stevia y del steviosido en humanos. Un comida estándar suplementada con $1 \mathrm{~g}$ de steviosido administrada a 12 sujetos diabéticos de tipo 2, es capaz de reducir los niveles de glucosa postprandial en sangre sobre un 18\% (Gregersen et al., 2004). Se observó un leve incremento en la circulación de insulina y un descenso en los niveles de glucagones. El índice insulinogénico indicó que tras el tratamiento con steviosido se incrementó un $40 \%$ la secreción de insulina. No se observó pérdida de glucosa urinaria por el consumo de steviosido, esto implica que el steviosido puede tener un efecto directo en la disposición de la insulina periférica inducido por la insulina responsable del descenso en los niveles en sangre de glucosa postprandial. Esto puede incluir un aumento en el almacenamiento de glucógeno en el hígado.

En contraste con lo anterior, Barriocanal et al. (2008), ha expuesto que el consumo prolongado de steviosido ( $>92 \%$ pureza) de $250 \mathrm{mg} 3$ veces/día durante 3 meses, cantidad similar a la usada como dulcificante, no tiene efectos farmacológicos en individuos diabéticos tanto de tipo 1 como de tipo 2 así como en sujetos normotensos o hipotensos. No se encontró menos glucosa en sangre o presión sanguínea. Se desconoce la explicación a esta carencia de efecto del steviosido, pero se debe señalar que el steviosido parece tener la habilidad de bajar los niveles de glucosa en plasma y la presión sanguínea sólo cuando estos parámetros son anormalmente elevados. Estos resultados son consecuentes con aquellos obtenidos previamente a corto plazo sobre los efectos en individuos sanos (Geuns et al., 2007).

El segundo componente más abundante de la hoja de stevia es el rebaudiosido A, que se diferencia del steviosido porque tiene una mitad de glucosa adicional. Esto eleva la posibilidad de que el rebaudiosido A pueda poseer efectos hipoglucémicos similares a los del steviosido y el extracto de stevia. De hecho, rebaudiosido A estimula la liberación de insulina considerablemente en ratones en presencia de grandes concentraciones de glucosa $(>6,6 \mathrm{mM})$ (Abudula et al., 2004). Inesperadamente, un estudio de tratamiento a largo plazo de ratas GK con rebaudiosido A fracasó en el estímulo de secreción de insulina. De manera parecida, el consumo a largo plazo de rebaudiosido A durante 16 semanas por parte de sujetos con diabetes mellitus tipo 2 no ha tenido efecto en la homeostasis de la glucosa, ni en la presión sanguínea (Maki et al., 2008).

Basado en estudio en células, animales y humanos, steviosido y sus compuestos relacionados (steviol y rebaudiosido A) afectan a la glucosa plasmática mediante la modulación de la secreción de insulina y la sensibilidad, lo que mejora la eliminación de glucosa en el plasma. También inhiben la absorción de glucosa intestinal y la generación de glucosa por el hígado alterando la actividad de varias enzimas claves envueltas en la síntesis de la glucosa reduciendo así el aporte de glucosa plasmática. Es interesante resaltar que los efectos del steviosido dependen enormemente de los niveles de glucosa plasmática, siendo observados sólo cuando estos son elevados. Así, parece ser totalmente seguro para individuos sanos. De todas formas, el mecanismo de este efecto no se conoce.

Se observó que los valores basales de creatinina no muestran diferencia significativa entre los tratamientos fluctuando los promedios de 0.6 a $0.65 \mathrm{mg} / \mathrm{dl}$ ( $\mathrm{p}>0.05$ ). A los 60 días no existe diferencia significativa en los tratamientos con extracto acuoso de Stevia rebaudiana $0.3,0.6$ y $0.9 \mathrm{~g} / \mathrm{kg} /$ día respecto al grupo control ( $\mathrm{p}>0.05)$. Por lo que podemos establecer que el extracto acuoso de Stevia rebaudina no provoca insuficiencia renal.

Se encontró a los 60 días de tratamiento una disminución significativa en el peso corporal de las ratas con tratamiento con extracto acuoso de Stevia rebaudiana $0.3,0.6$ y $0.9 \mathrm{~g} /$ $\mathrm{kg} /$ día respecto al grupo control $(\mathrm{p}<0.05)$. Estos resultados coinciden con lo reportado por (Jeppesen et al., 2000; Lailerd et al., 2004; JECFA,2006). El mecanismo probable es que el esteviol disminuye la absrción de glucosa a nivel intestinal y el esteviosido incrementa la secreción de insulina y aumenta la sensibilidad al mismo (Jeppesen et al., 2000; Lailerd et al., 2004).

En el diagnóstico histopatológico de Higado, riñón y estomago de Rattus norvegicus variedad Sprague Dawley con tratamiento de extracto acuoso de Stevbia rebaudiana en dosis de $0.3,0.6$ y $0.9 \mathrm{~g} / \mathrm{kg} /$ día no se encontró alteración microscópica. Estos resultados coinciden con lo reportado por (Akashi \& Yokoyama, 1975; Xili et al., 1992; Toskulkao 
et al.,1997).

En estudios de vitro que implican varias líneas de célula diferentes de riñón e intestino indicaron que steviosido en una concentración tan alta como $2 \mathrm{mM}$ tienen que ser expuestos a células para afectar la viabilidad de célula, pero $0.2 \mathrm{mM}$ de steviol reduce significantemente la viabilidad de la célula (Srimaroeng et al., 2005b;Boonkaewwan et al., 2008). Este efecto tóxico puede ser consecuencia de una interrupción del metabolismo mitocondrial como se ha sugerido en estudios anteriores (Toskulkao et al., 1997).

En evaluaciones de toxicidad agudas y crónicas de ingestión steviosido investigado en ratones, ratas y hámsteres, con un consumo de steviosido tan elevado como $15 \mathrm{~g} / \mathrm{Kg}$ de peso corporal no produce toxicidad aguda alguna (Akashi \& Yokoyama, 1975; Xili et al., 1992; Toskulkao et al.,1997). Sin embargo, la administración oral de steviol es letal con un LD50 de 5-20 g/ $/ \mathrm{kg}$ de peso corporal, dependiendo de la especie animal, siendo los hámsteres más susceptibles a la toxicidad del steviol que las ratas o los ratones (Toskulkao et al.,1997). El examen histológico del tratamiento con steviol en hámsteres ha revelado la degeneración de la célula tubular del proximal, la extensión de la cual esta relacionada con el la subida en el suero sanguíneo del nitrógeno de urea y la creatinina, indicadores del deterioro de la función renal. Así pues, la causa de muerte en hámsteres tratados con steviol es un fallo renal agudo.

La ingesta de steviosido ( $750 \mathrm{mg} /$ día durante 3 meses) en individuos sanos o con enfermedades subyacentes como la diabetes mellitus e hipertensión, no produce efectos adversos o anormalidades en el hígado o en la función renal (Barriocanal et al., 2008). De acuerdo con estudios farmacocinéticos previos en humanos, después de la administración oral de una dosis individual de 4,2 $\mathrm{mg}$ de steviosido por $\mathrm{Kg}$ de peso corporal, supone la máxima concentración de steviol glucorinido y steviol libre en plasma de $1,89 \mu \mathrm{g} / \mathrm{ml}(3,7 \mu \mathrm{M})$ y $0,19 \mu \mathrm{g} / \mathrm{ml}(0,38 \mu \mathrm{M})$ respectivamente (Wheeler et al., 2008). Este nivel de steviol, no debería ser tóxico para las células humanas, y parece que el steviol glucorónido no se convierte de nuevo en steviol.

\section{REFERENCIAS BIBLIOGRÁFICAS}

1. Abudula, R., Jeppesen, P. B., Rolfsen, S. E.D., Xiao, J.,\&Hermansen, K. (2004). Rebaudioside A potently stimulates insulin secretion from isolated mouse islets: studies on the dose-, glucose-, and calciumdependency. Metabolism 53(10), 1378-1381.

2. Akashi, H., \& Yokoyama, Y. (1975). Security of driedleaf extracts of stevia: toxicological tests. Food Industry $18,34-43$.

3. Barriocanal, L. A., Palacios, M., Benitez, G., Benitez, S., Jimenez, J. T., Jimenez, N (2008). Apparent lack of pharmacological effect of steviol glycosides used as sweeteners in humans. A pilot study of repeated exposures in some normotensive and hypotensive individuals and in Type 1 and Type 2 diabetics. Regul Toxicol Pharmacol 51(1), 37-41.

4. Boeckh, E. M. A., \& Humboldt, G. (1981). Efeitos cardiocirculatorios do extrato aquoso total em individuos normais e do esteviosideo em ratos. Cienc Cult 32, 208-210.

5. Boonkaewwan, C., Ao, M., Toskulkao, C., \& Rao, M. C. (2008). Specific immunomodulatory and secretory activities of stevioside and steviol in intestinal cells. $\mathbf{J}$ Agric Food Chem 56(10), 3777-3784.

6. Boonkaewwan, C., Toskulkao, C., \& Vongsakul, M. (2006). Anti-inflammatory and immunomodulatory activities of stevioside and its metabolite steviol on THP-1 cells. J Agric Food Chem 54(3), 785-789.

7. Cardoso, V. N., Barbosa, M. F., Muramoto, E., Mesquita, C. H., \& Almeida, M. A. (1996). Pharmacokinetic studies of 131I-stevioside and its metabolites. Nucl Med Biol 23(1), 97-100.

8. Chan, P., Tomlinson, B., Chen, Y. J., Liu, J. C., Hsieh, M. H., \& Cheng, J. T. (2000). A doubleblind placebocontrolled study of the effectiveness and tolerability of oral stevioside in human hypertension. Br J Clin Pharmacol 50(3), 215-220.

9. Ferri, L. A. F., Alves-Do-Prado,W., Yamada, S. S., Gazola, S., Batista, M. R., \& Bazotte, R. B. (2006). Investigation of the antihypertensive effect of oral crude stevioside in patients with mild essential hypertension. Phytother Res 20(9), 732-736.

10. Gardana, C., Simonetti, P., Canzi, E., Zanchi, R., \& Pietta, P. (2003). Metabolism of stevioside and rebaudioside A from Stevia rebaudiana extracts by human microflora. J Agric Food Chem 51(22), 6618-6622.

11. Geuns, J. M., Bruggeman, V., \& Buyse, J. G. (2003). Effect of stevioside and steviol on the developing broiler embryos. J Agric Food Chem 51(17), 5162-5167.

12. Hanson, J. R., \& De Oliveira, B. H. (1993). Stevioside and related sweet diterpenoid glycosides. Nat Prod Rep 10(3), 301-309.

13. Hutapea, A. M., Toskulkao, C., Buddhasukh, D., Wilairat, P., \& Glinsukon, T. (1997). Digestion of stevioside, a natural sweetener, by various digestive enzymes. J Clin Biochem Nutr 23(3), 177-186.

14. JECFA, Joint FAO/WHO Expert Committee on Food Additives,, (2006). Steviol Glycosides [Addendum to stevioside]. In: Safety Evaluation of Certain Food Additives: Sixtythird

15. Jeppesen, P. B., Gregersen, S., Alstrup, K. K., \& Hermansen, K. (2002). Stevioside induces antihyperglycaemic, insulinotropic and glucagonostatic effects in vivo: studies in the diabetic Goto-Kakizaki (GK) rats. Phytomedicine 9(1), 9-14.

16. Jeppesen, P. B., Gregersen, S., Rolfsen, S. E. D., Jepsen, M., Colombo, M., Agger, A., et al. (2003). Antihyperglycemic and blood pressure-reducing effects of stevioside in the diabetic Goto-Kakizaki rat. Metabolism 52(3), 372-378.

17. Kinghorn, A. D., \& Soejarto, D. D. (1985). Current status of stevioside as a sweetening agent for human use. In H. Wagner, H. Hikino, \& N. R. Farnsworth (Eds.), Economic and medical plant research Vol. 1 (pp. 1-52). London: Academic Press.

18. Kitahata, S., Ishikawa, H., Miyata, T., \& Tanaka, O. (1989). Production of rubusoside derivatives by transgalactosylation of various $\beta$-galactosidases. Agric Biol Chem 53 (11), 2923-2928.

19. Koyama, E., Kitazawa, K., Ohori, Y., Izawa, O., Kakegawa, K., Fujino, A., et al. (2003). In vitro metabolism of the glycosidic sweeteners, stevia mixture and enzymatically modified stevia in human intestinal 
microflora. Food Chem Toxicol 41(3), 359-374.

20. Maki, K. C., Curry, L. L., Reeves, M. S., Toth, P. D., McKenney, J. M., Farmer, M. V., et al. (2008). Chronic consumption of rebaudioside $\mathrm{A}$, a steviol glycoside, in men and women with type 2 diabetes mellitus. Food Chem Toxicol 46(7 Suppl 1), S47-53.

21. Matsui, M., Matsui, K., Kawasaki, Y., Oda, Y., Noguchi, T., Kitagawa, Y., et al. (1996). Evaluation of the genotoxicity of stevioside and steviol using six in vitro and one in vivo mutagenicity assays. Mutagenesis 11(6), 573-579.

22. Melis, E. (1997). Effects of steviol on renal function and mean arterial pressure in rats. Phytomedicine 3, 349-352.

23. Pezzuto, J. M., Compadre, C. M., Swanson, S. M., Nanayakkara, D., \& Kinghorn, A. D. (1985). Metabolically activated steviol, the aglycone of stevioside, is mutagenic.Proc Natl Acad Sci U S A 82(8), 2478-2482.

24. Planas, G. M., \& Kuc, J. (1968). Contraceptive properties of Stevia rebaudiana. Science 162 (3857), 1007.

25. Roberts, A., \& Renwick, A. G. (2008). Comparative toxicokinetics and metabolism of rebaudioside A, stevioside, and steviol in rats. Food Chem Toxicol 46(7 Suppl 1), S31-39.

26. Sekihashi, K., Saitoh, H., \& Sasaki, Y. (2002). Genotoxicity studies of stevia extract and steviol by the comet assay. J Toxicol Sci 27(Suppl 1), 1-8.

27. Shibata, H., Sawa, Y., Oka, T., Sonoke, S., Kim, K. K., \& Yoshioka, M. (1995). Steviol and steviol-glycoside: glucosyltransferase activities in Stevia rebaudiana Bertoni - purification and partial characterization. Arch Biochem Biophys 321(2), 390-396.

28. Shiotsu, S. (1996). Fertility study of Stevia decoction in rats. Tech J Food Chem. Chemicals 4, 108-113.

29. Shiozaki, K., Fujii, A., Nakano, T., Yamaguchi, T., \& Sato, M. (2006). Inhibitory effects of hot water extract of the Stevia stem on the contractile response of the smooth muscle of the guinea pig ileum. Biosci Biotechnol Biochem 70(2), 489-494.

30. Suttajit, M., Vinitketkaumnuen, U., Meevatee, U., \& Buddhasukh, D. (1993). Mutagenicity and human chromosomal effect of stevioside, a sweetener from Stevia rebaudiana Bertoni. Environ Health Perspect 101(Suppl 3), 53-56.

31. Toskulkao, C., Deechakawan, W., Leardkamolkarn, V., Glinsukon, T., \& Buddhasukh, D. (1994). The low calorie natural sweetener stevioside: nephrotoxicity and its relationship to urinary enzyme excretion in the rat. Phytother Res 8(5), 281-286.

32. Toskulkao, C., Sutheerawattananon, M., \& Piyachaturawat, P. (1995). Inhibitory effect of steviol, a metabolite of stevioside, on glucose absorption in everted hamster intestine in vitro. Toxicol Lett $80(1-3)$, 153-159.

33. Wheeler, A., Boileau, A. C., Winkler, P. C., Compton, S. C., Prakash, J., Jiang, X., et al. (2008). Pharmacokinetics of rebaudioside $\mathrm{A}$ and stevioside after single oral doses in healthy men. Food Chem Toxicol 46, S54-S60.

34. Wingard, R. E., Jr., Brown, J. P., Enderlin, F. E., Dale, J. A., Hale, R. L., \& Seitz, C. T. (1980). Intestinal degradation and absorption of the glycosidic sweeteners stevioside and rebaudioside A. Experientia 36(5), 519-520.

35. Xili, L., Chengjiany, B., Eryi, X., Reiming, S., Yuengming, W., Haodong, S., et al. (1992). Chronic oral toxicity and carcinogenicity study of stevioside in rats. Food Chem Toxicol 30(11), 957-965.

36. Yasukawa, K., Kitanaka, S., \& Seo, S. (2002). Inhibitory effect of stevioside on tumor promotion by 12-O-tetradecanoylphorbol-13-acetate in two-stage carcinogenesis in mouse skin. Biol Pharm Bull 25(11), 1488-1490. 